\title{
Breast Volume Calculation Using a Low-Cost Scanning System
}

\author{
Simon B. CHOPPIN ${ }^{a}$, Heidi PROBST ${ }^{b}$, Amit GOYAL ${ }^{c}$, Sean CLARKSON ${ }^{a}$, Jonathan WHEAT ${ }^{a}$ \\ ${ }^{a}$ Centre for Sports Engineering Research, Sheffield Hallam University, Sheffield, UK; \\ ${ }^{\mathrm{b}}$ Centre for Health and Social Care Research, Sheffield Hallam University, Sheffield, UK; \\ 'Derby Hospitals, Derby, UK \\ http://dx.doi.org/10.15221/13.012
}

\begin{abstract}
Breast volume has been identified as a key metric in assessing patients for reconstructive surgery. Scanning systems have measured breast volume but they have tended to rely on expensive hardware and software. This paper discusses the development and assessment of an algorithm capable of calculating breast volume from 3D point data. A mannequin was scanned (using a custom, Kinect based scanning system) with one of two breast prostheses attached $-400 \mathrm{~g}$ or $600 \mathrm{~g}$. Each scan was assessed by three independent operators: seven anatomical points were identified representing the boundary of the breast region, which was then isolated. A Coons patch was used to represent the invisible chest surface lying below the breast tissue. A trapezium rule based approach was used to calculate the volume of the enclosed region between the breast and chest surfaces. Breast volume was over-estimated by $130 \mathrm{cc}$ with the $400 \mathrm{~g}$ prosthesis $(30.3 \%)$ and $206 \mathrm{cc}(33.3 \%)$ with the $600 \mathrm{~g}$ prosthesis, suggesting positive proportional bias. Average reliability was $\pm 59.7 \mathrm{cc}$ for the $400 \mathrm{~g}$ prosthesis $(13.9 \%)$ and $\pm 34.7 \mathrm{cc}$ for the $600 \mathrm{~g}$ prosthesis $(5.6 \%)$ - approaching the levels required to differentiate between implant sizes $(25-50 \mathrm{cc})$. Future work will focus on refining the hardware and software of this scanning system - minimising proportional basis and maximising reliability of measurement.
\end{abstract}

Keywords: $3 \mathrm{~d}$ body scanning, proceedings, format

\section{Introduction}

Three dimensional (3D) scanning technologies provide non-contact measurements of anatomical surfaces. Surgeons have used them to plan and evaluate surgery, for example, in cephalometrics $[1,2]$.

In breast surgery, Tepper et al. [3] recently introduced the concept of mammometrics, a method of breast measurement using 3D points and planes. Lui et al. [4] evaluated 3D surface imaging to measure breast asymmetry, suggesting that measurement technologies are important for optimal implant selection. While the benefits of accurate breast measurement are tangible, current commercial systems are prohibitively expensive and not in widespread use. For this reason manual measurements are often used to plan reconstructive surgery and choose implant size. The disadvantages of this approach are reflected in a quote from Tepper et al. [3]

"...the overall success of breast reconstruction is limited by the inability of plastic surgeons to objectively assess breast volume and shape, which may result in suboptimal outcomes."

This is reflected in the UK's National Mastectomy and Breast Reconstruction Audit (2010) which showed that 1 in 10 mastectomy patients and 1 in 6 reconstruction patients required additional surgery [5].

Recent consumer 'depth cameras' such as the Microsoft Kinect provide 3D point data at low cost around £200. A prototype breast measurement system - using two Kinects - was used to measure straight line and surface distances between key anatomical landmarks for breast surgery (see also Oliveira et al.[6]). The system compared well with manual measurements [7] and a similar study [8] which used a commercial scanner. However, this system is limited in that it does not include a routine to calculate breast volume, a metric of primary importance [9-12].

Authors have made measurements of breast volume using commercial laser $[9,11,12]$ (Konica Minolta vivid 910), commercial stereo photogrammetric [13] (3dMD), custom structured light [14] and custom stereo photogrammetric [15] systems (for a thorough review of technological approaches to aesthetic evaluation in breast surgery, see Oliveira et al. [16]). In each case the same two challenges were addressed: how to identify the extremities of the breast region and how to identify the chest wall lying below the scanned breast surface. All authors identified the extremity of the breast region using a set of manually identified points. In some cases these were anatomically identified $[9,11-14]$ but in others the operator made personal judgments regarding breast extremity [15]. In every case the system operator identified points using the scanned video image rather than identifying points on the patient prior to scanning. The techniques used to join the selected points and create a continuous boundary 
were not clearly described although most authors use a parameterised surface known as a 'Coons patch' $[17,18]$ to define the invisible chest wall boundary. Not only are the methods described inconsistent, between authors but the routines used may rely on expensive commercial software.

There is a need to develop volume measurement techniques which do not rely on expensive, hence prohibitive, software and hardware and also assess the accuracy of these techniques to determine their usefulness to a clinical practitioner.

Therefore, this study had two objectives,

1. To develop algorithms capable of measuring breast volume from 3D point cloud data - these should not be reliant on 'black-box' commercial software routines

2. To assess the accuracy and reliability of the acquired volume measurement

\section{Methods}

This project consisted of three stages

1. Acquisition of point cloud data

2. Development of volume algorithm

3. Assessment of accuracy and reliability

Each stage will be considered in turn

\subsection{Acquisition of point cloud data}

A male mannequin with attached breast prostheses (the volume of a prosthetic breast can be measured simply through water displacement) was used as a model. Two breast sizes were used, a small $400 \mathrm{~g}$ prosthetic and a medium/large $600 \mathrm{~g}$ prosthetic, shown in figure 1.

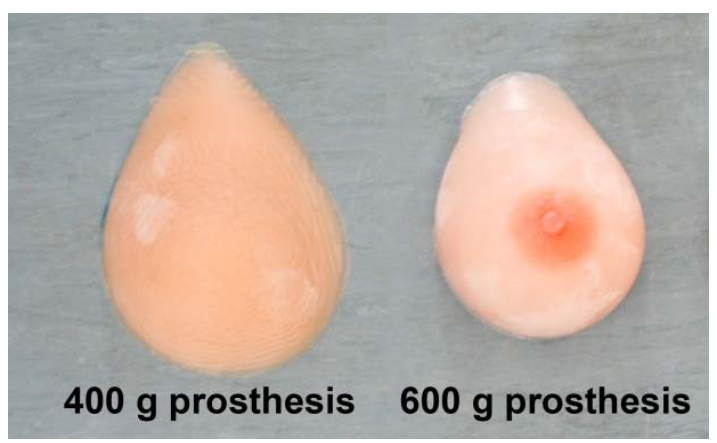

Figure 1. The 400 and 600 gram (429 and 618 cubic centimetre) prostheses used in the study.

The model was scanned using three, extrinsically calibrated, Microsoft Kinect depth cameras. The cameras were orientated to capture the left, right and underside of the attached prosthesis at a distance of approximately $0.7 \mathrm{~m}$. The general set-up is shown in figure 2 .

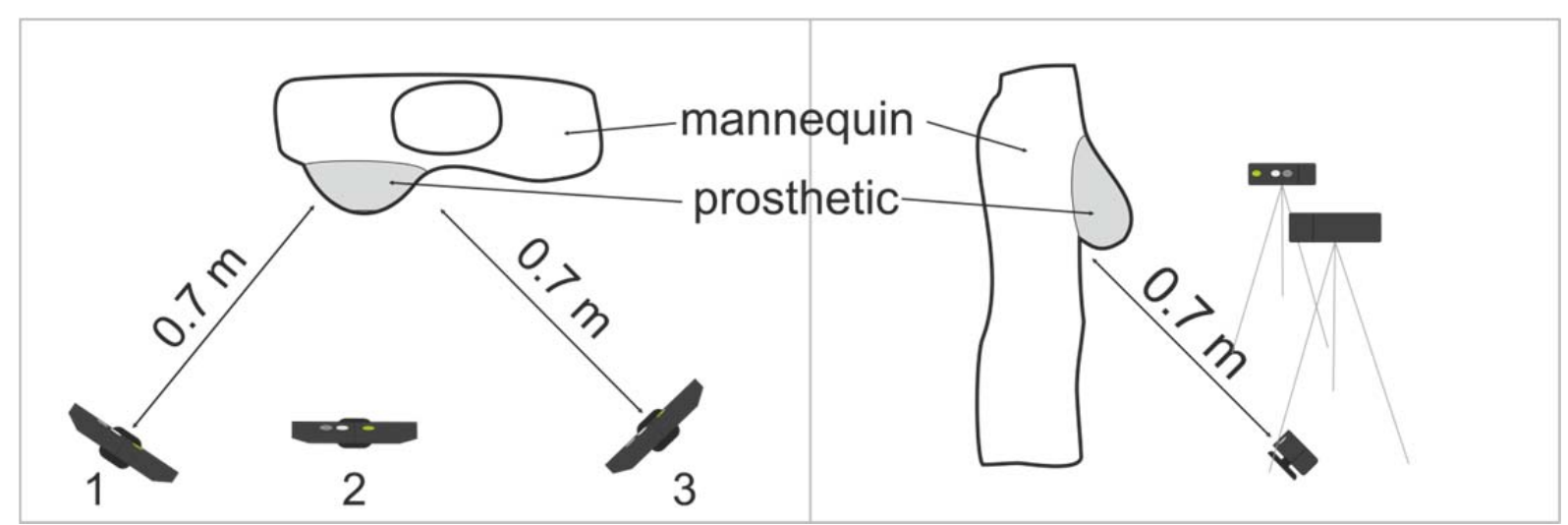

Figure 2. The approximate location of the three Kinect system used to capture the breast model.

The Kinect system was extrinsically calibrated using two polystyrene spheres on a metal rod - figure 3 . The spheres were moved into 20 different positions within the scanning space (nominally occupied by the model). The sphere centre locations were identified using a combination of computer vision (sobel edge detection and Hough transform) and optimisation techniques. These centre locations gave the relative orientation and translation of each Kinect through singular value decomposition techniques $[19,20]$ - a RANSAC algorithm was used to discard outliers. 


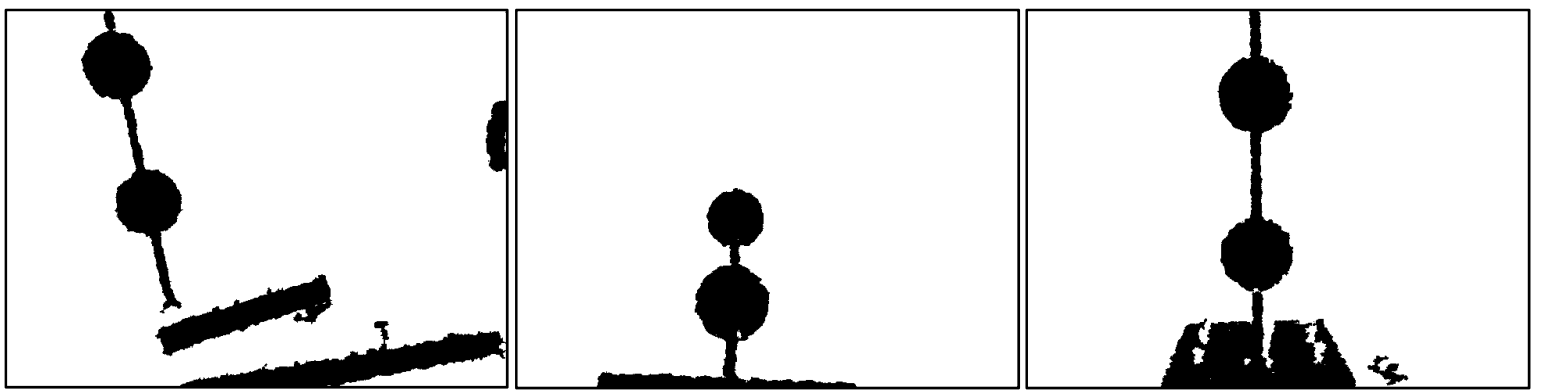

Figure 3. The calibration object, as viewed by each Kinect's depth camera, presented as a binary image.

At collection, each Kinect scanned the model in sequence, with the projectors of the other two Kinect's turned off. The entire scanning sequence took around one second. Each point cloud was transformed to a global reference frame - the local axes set of Kinect number 1 (figure 2).

Two combined point cloud sets were obtained, one for each of the breast prostheses - these were used in combination with a processing algorithm to calculate breast volume.

\subsection{Breast volume calculation algorithm}

The software routines were developed in Matlab 2013a (Mathworks, USA). The process involved four discrete stages

1. Identification of points on breast boundary

2. Joining of points to form continuous breast boundary

3. Creation of chest-wall surface

4. Calculation of volume

\subsubsection{Identification of point on breast boundary}

Seven discrete, anatomically derived points were chosen to represent the boundary of the breast region - these were based on the work by Kovacs et al. [9] and are presented in figure 4 and table 1. A graphical user interface (GUI) displayed the combined point data, allowing a user to define the location of each point with a mouse click. Once each point had been defined, they were joined in sequence to form a continuous boundary.

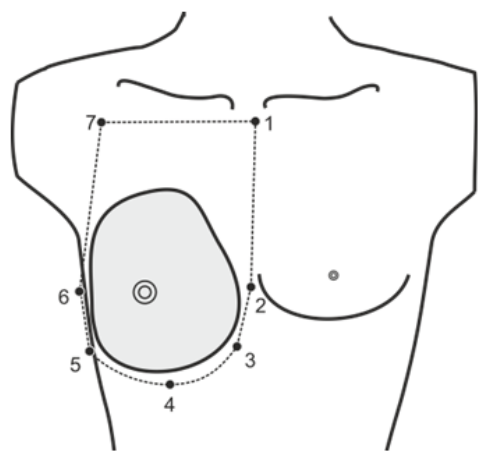

Figure 4. The seven points chosen to represent the breast boundary.

Table 1. A description of the point locations used to define the breast boundary.

\begin{tabular}{|c|c|}
\hline Point & Location \\
\hline 1. Sternal notch: & The visible dip at the base of the neck, between the clavicles. \\
\hline 2. Medial breast: & $\begin{array}{l}\text { A point in line with the nipple, approximately } 1-2 \mathrm{~cm} \text { from the medial extent } \\
\text { of the breast. }\end{array}$ \\
\hline 3. Medial infra mammary fold(IMF) & A point approximately $1-2 \mathrm{~cm}$ from the medial extent of the IMF \\
\hline 4. Bottom of IMF: & A point approximately $1-2 \mathrm{~cm}$ below the bottom of the IMF \\
\hline 5. Lateral IMF: & A point approximately $1-2 \mathrm{~cm}$ from the lateral extent of the IMF \\
\hline 6. Lateral breast: & $\begin{array}{l}\text { A point in-line with the nipple, approximately } 1-2 \mathrm{~cm} \text { from the lateral extent } \\
\text { of the breast }\end{array}$ \\
\hline 7. Clavicle: & A point near the lateral extent of the clavicle, at the same height as point 1 \\
\hline
\end{tabular}




\subsubsection{Joining of points to form continuous breast boundary}

Points 1-2, 6-7 and 7-1 were joined with a line running along the model's scanned surface. The line chosen was the shortest (between the terminal points) which travelled along the model's surface and existed within a single plane. The methodology is described by Wheat et al. [7]. Points 2-3-4-5-6 were joined with a spline to form the curve lying underneath the infra-mammary-fold (IMF). To ensure that the line lay on the model's surface, interpolated spline points were replaced with the closest corresponding point on the model's scanned surface. An illustration of selected points before and after 'joining' is shown in figure 5 .
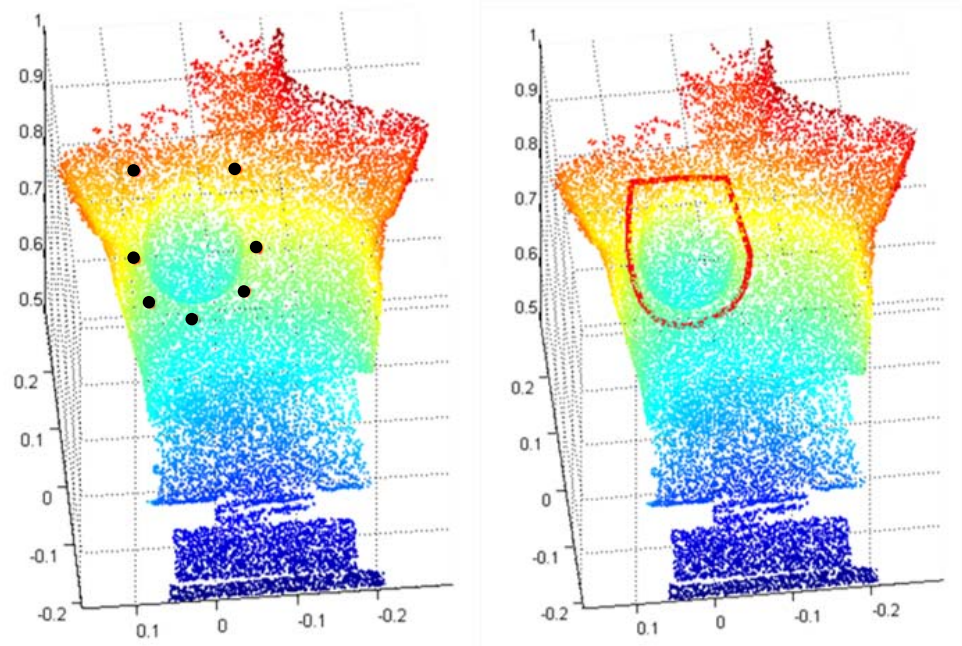

Figure 5. The model's scanned surface showing seven anatomical points and the result of the joining process, taken from the Matlab GUI.

\subsubsection{Creation of chest wall surface}

Once the extremities of the breast region were identified, the breast was isolated. In order to create a closed volume, a chest surface was created using a Coons patch [17], a doubly curved surface previously used for this application $[11,13,15]$. The lines created in the previous step were used as boundary curves, with the necessary processing executed using free routines provided by Spink [21]. Once created, the patched chest surface was interpolated to create a number of discrete points which in combination with the breast points, enclose the breast volume, as shown in figure 6 .

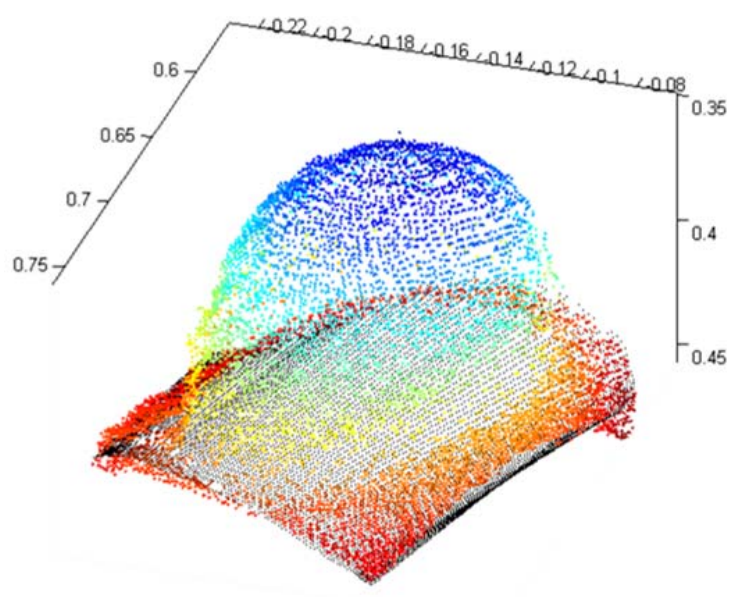

Figure 6. The isolated breast, showing the interpolated chest surface.

\subsubsection{Calculation of volume}

To calculate volume the breast points were divided into 100 equal slices - figure $7 \mathrm{a}$. Within each slice the area underneath the exterior 'breast' and interior 'chest' points was calculated using the trapezium rule - figure $7 \mathrm{~b}$. The difference in area between these two regions was deemed to be the enclosed breast area. The volume of breast contained within each slice was calculated by multiplying the enclosed breast area by the width of each slice. The summed volume of all slices gave the breast volume. 


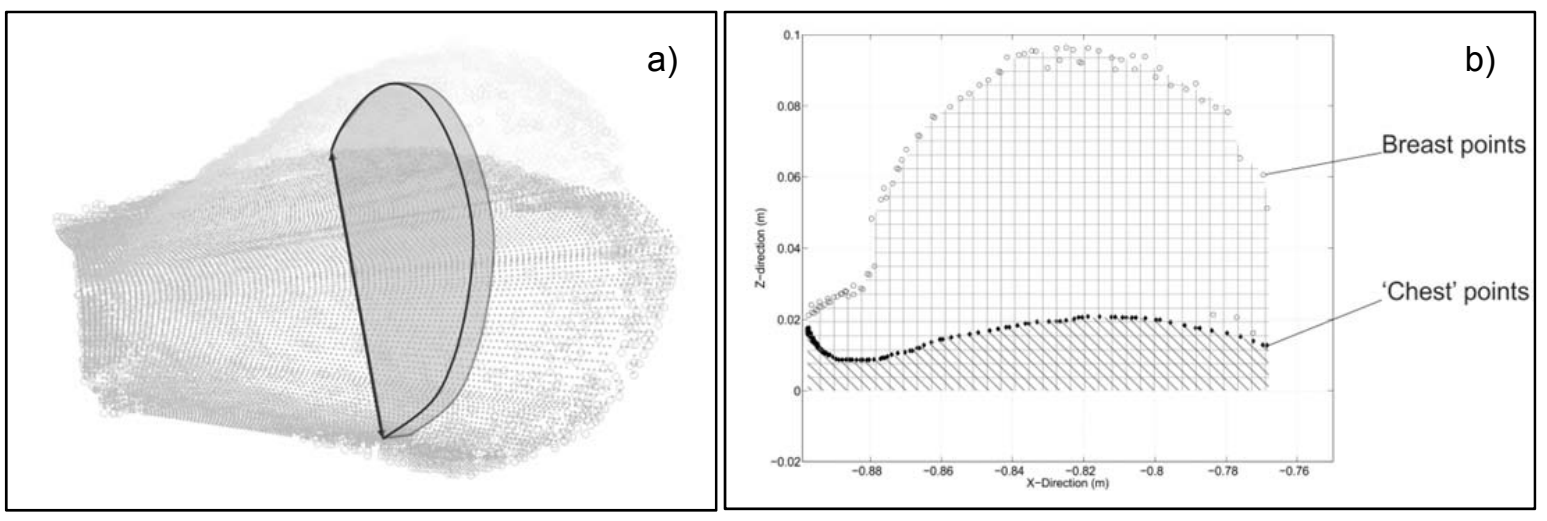

Figure 7. The methodology used to calculate breast volume.

\subsection{Assessment of accuracy and reliability}

To assess the absolute accuracy and reliability of the method, breast volumes given by the calculation algorithm were compared with volumes measured using a water displacement technique (based on the Archimedes' principle [22]). Three independent operators were used to assess the algorithm's reliability. Each operator was given a software tutorial and the information shown in figure 4 and table 1. The entire process was repeated five times for each of the two breast prostheses used in this study. The following statistical measures were evaluated:

- Absolute accuracy - the difference between breast volumes given by the software and water displacement.

- Reliability - the deviation of each operator from their average reported breast volume.

- Differential accuracy - How accurately the difference in volume between the two breast prostheses was reported.

Note, breast volume of prostheses on a flat surface: The entire process was repeated with each breast prostheses placed on a flat surface (the laboratory floor). The purpose of this process was to assess how the mannequin itself may influence the reported breast volume. Only a single operator was used in this case, with five repeated measures.

\section{Results}

\subsection{Breast volumes reported using water displacement}

Table 2 shows the volume of each prosthesis in cubic centimetres (cc).

Table 2. The volume of each breast prosthesis as measured by the water displacement method

\begin{tabular}{lll}
\hline \multicolumn{3}{l}{ Water Displacement } \\
\hline $400 \mathrm{~g}$ & $600 \mathrm{~g}$ & Difference \\
429 & 618 & 189 \\
\hline
\end{tabular}

\subsection{Breast volumes reported using software algorithms}

The software algorithm systematically over-estimated breast volume compared to water displacement - table 3 - even when assessment was made with the breasts on a flat surface.

Table 3. The reported breast volumes as measured by three independent operators and with the prostheses placed on a flat surface. All volume measures reported in cubic centimetres (cc). The final row shows the mean volume measure with the standard deviation in parentheses.

\begin{tabular}{llllllll}
\hline \multicolumn{2}{c}{ Operator 1 } & \multicolumn{2}{c}{ Operator 2 } & \multicolumn{2}{c}{ Operator 3 } & \multicolumn{2}{c}{ Floor } \\
\hline $400 \mathrm{~g}$ & $600 \mathrm{~g}$ & $400 \mathrm{~g}$ & $600 \mathrm{~g}$ & $400 \mathrm{~g}$ & $600 \mathrm{~g}$ & $400 \mathrm{~g}$ & $600 \mathrm{~g}$ \\
554 & 794 & 557 & 825 & 508 & 812 & 500 & 674 \\
539 & 788 & 607 & 821 & 513 & 816 & 553 & 669 \\
656 & 751 & 479 & 814 & 522 & 836 & 480 & 720 \\
530 & 724 & 503 & 856 & 682 & 928 & 481 & 675 \\
488 & 808 & 618 & 874 & 629 & 923 & 514 & 662 \\
$\mathbf{5 5 3 ( 6 2 )}$ & $\mathbf{7 7 3 ( 3 5 )}$ & $\mathbf{5 5 3 ( 6 2 )}$ & $\mathbf{8 3 8 ( 2 6 )}$ & $\mathbf{5 7 1}(80)$ & $\mathbf{8 6 3 ( 5 8 )}$ & $\mathbf{5 0 6 ( 3 0 )}$ & $\mathbf{6 8 0}(23)$ \\
\hline
\end{tabular}




\subsection{Absolute accuracy}

Accuracy was greatest when the breast was assessed on a flat surface. Differences between operators were observed, although a t-test revealed them to be non-significant on average - table 1 . With the mannequin, breast volume was over-estimated by $130 \mathrm{cc}$ with the $400 \mathrm{~g}$ prosthesis $(30.3 \%)$ and $206 \mathrm{cc}(33.3 \%)$ with the $600 \mathrm{~g}$ prosthesis, suggesting positive proportional bias. This effect is reversed on a flat surface, $77 \mathrm{cc}$ over estimation with the $400 \mathrm{~g}$ prostheses (17.9\%) and $62 \mathrm{cc}$ over estimation with the $600 \mathrm{~g}$ prosthesis (10\%).

Table 4. The difference between volume measures reported by the software and those measured using water displacement, in cubic centimetres. The final row shows the mean volume difference measure with the standard deviation in parentheses.

\begin{tabular}{llllllll}
\hline \multicolumn{2}{c}{ Operator 1 } & \multicolumn{2}{c}{ Operator 2 } & \multicolumn{2}{c}{ Operator 3 } & \multicolumn{2}{c}{ Floor } \\
\hline $400 \mathrm{~g}$ & $600 \mathrm{~g}$ & $400 \mathrm{~g}$ & $600 \mathrm{~g}$ & $400 \mathrm{~g}$ & $600 \mathrm{~g}$ & $400 \mathrm{~g}$ & $600 \mathrm{~g}$ \\
125 & 176 & 128 & 207 & 79 & 194 & 71 & 56 \\
110 & 170 & 178 & 203 & 84 & 198 & 124 & 51 \\
227 & 133 & 50 & 196 & 93 & 218 & 51 & 102 \\
101 & 106 & 74 & 238 & 253 & 310 & 52 & 57 \\
59 & 190 & 189 & 256 & 200 & 305 & 85 & 44 \\
$\mathbf{1 2 4}(62)$ & $\mathbf{1 5 5 ( 3 5 )}$ & $\mathbf{1 2 4}(62)$ & $\mathbf{2 2 0 ( 2 6 )}$ & $\mathbf{1 4 2 ( 8 0 )}$ & $\mathbf{2 4 5 ( 5 8 )}$ & $\mathbf{7 7 ( 3 0 )}$ & $\mathbf{6 2 ( 2 3 )}$ \\
\hline
\end{tabular}

\subsection{Reliability}

The standard error of measurement was approximated by dividing the standard deviation measure in table 3 by $\sqrt{n}$ (2.24), a 95\% confidence interval was assumed to be the standard error multiplied by 1.96. The measurements of the larger, $600 \mathrm{~g}$ prosthetic were shown to be more reliable - table 5.

Table 5. The reliability of measurement obtained from the standard deviation values in table $3-$ given in cubic centimetres.

\begin{tabular}{llllllll}
\hline \multicolumn{2}{c}{ Operator 1 } & \multicolumn{2}{c}{ Operator 2} & \multicolumn{2}{c}{ Operator 3 } & \multicolumn{2}{c}{ Floor } \\
\hline $400 \mathrm{~g}$ & $600 \mathrm{~g}$ & $400 \mathrm{~g}$ & $600 \mathrm{~g}$ & $400 \mathrm{~g}$ & $600 \mathrm{~g}$ & $400 \mathrm{~g}$ & $600 \mathrm{~g}$ \\
\pm 55 & \pm 30 & \pm 54 & \pm 23 & \pm 70 & \pm 51 & \pm 26 & \pm 20 \\
\hline
\end{tabular}

\subsection{Differential accuracy}

Average differential accuracy is easily obtained from table 3 , the standard deviation of this measurement was calculated using the 25 possible combinations of volume measurements from each operator. The values are shown in table 6.

Table 6. The mean reported differences in breast volume from each operator, as measured by the software, given in cubic centimetres.

\begin{tabular}{cccc}
\hline Operator 1 & Operator 2 & Operator 3 & Floor \\
\hline $220(65)$ & $285(61)$ & $292(90)$ & $174(35)$ \\
\hline
\end{tabular}

\section{Discussion}

This report details the development of a breast volume calculation algorithm for use on point cloud data, the accompanying analysis indicates the relevance of this algorithm in a clinical setting. The motivation for this study was to develop algorithms which do not rely on expensive, commercial software packages and can process data obtained from low-cost scanning systems - giving wider access to the rich measurements made possible with 3D point data.

Breast volume was measured in two conditions, with the prostheses attached to a mannequin and with the prostheses placed on a flat surface. The mannequin condition was used to create a realistic testing environment for the volume algorithms. The floor condition was intended to be purposefully favourable, with a flat chest wall. Given this, it was no surprise that absolute accuracy and reliability were higher in the flat surface condition.

To this extent the floor condition tested the feasibility of the approach, a validation that the algorithm reports volume measures within usable bounds. There were many possible sources of error in volume calculation, the accuracy of the chest wall surface, the effect of breast boundary point location and the 
accuracy of the scanned point data. The flat surface condition was intended to control as many of these variables as possible and assess the efficacy of the volume algorithm itself (as far as possible given our scanning system).

While significant differences between operators were not found, large errors in individual scans are apparent from table 4 . Between all operators, accuracy ranged from $11.7 \%$ to $59.0 \%$ over-estimation. While the statistical power of these analyses isn't sufficient to draw specific conclusions about individual operators, it is apparent that significant errors can arise from poor point selection. In this experiment error was compounded by the type of mannequin used - an 'anatomically ideal' male. The pronounced pectoral muscles were almost certainly included within the measured breast tissue. I.e. the Coons patch enclosed the prosthetic breast and the mannequin's pectoral muscle - increasing the measure of volume. This explains the large, systematic over-estimation recorded by all operators, and the difference between the mannequin and flat surface conditions.

The algorithm calculates volume as the enclosed region between two surfaces. The external surface consists of the scanned breast surface and is subject to errors due to scanning accuracy. The internal surface is created using a Coons patch and is dependent on the location of the points selected by the operator. The algorithm assumes that the Coons patch and chest surface are congruent. In other words, the volume between the chest and patch will be zero - only breast tissue will be measured. This is not the case; chest surface which is not part of the breast was usually included in the volume calculation. In reality the boundary between the chest and breast region is difficult to detect and define using only visual techniques, particularly at the superior breast boundary. This difficulty is reflected in the variety of approaches chosen by other authors [9-15].

For the purposes of clinical assessment, reliability of measurement is perhaps of greater importance than absolute accuracy. The ability to confidently measure differentials in volume and reliably assess volume measures (even with a systematic offset) is essential when choosing surgical procedures and implant sizes. The differentials in volume between implant sizes are around $25-50 \mathrm{cc}$. For the mannequin, average reliability was $\pm 59.7 \mathrm{cc}$ for the $400 \mathrm{~g}$ prosthesis $(13.9 \%)$ and $\pm 34.7 \mathrm{cc}$ for the 600 g prosthesis $(5.6 \%)$ - approaching the levels required to differentiate between implant sizes. The observed proportional bias - which resulted in larger absolute error for the $600 \mathrm{~g}$ prosthesis - is a problem when assessing volume. Proportional error renders measures of differential volume increasingly inaccurate as breast size increases. This is a problem when measuring volume differences between a pre- and post-operative breast for example.

Previous research using commercial systems has achieved comparatively high levels of accuracy. Losken et al. Measured breast volume from 3D scans and compared this with the value obtained from water displacement (post mastectomy). From 19 participants, $85 \%$ of scanned volume measures were within $10 \%$ of those obtained through water displacement. Kovacs et al. [9] measured differential breast volume, pre- and post-surgery (both augmentation and reduction). The calculated scanned volume change differed from the 'operative' volume change by -2.79 cc \pm 11.76 in augmentation patients and $-0.04 \mathrm{cc} \pm 29.7 \mathrm{cc}$ in reduction patients. Our study has demonstrated levels of reliability within the bounds demonstrated by Losken et al. but with considerably higher systematic error reducing this should be the focus of future studies.

The scanning apparatus used in this project contributes to the reported errors in volume. A custom, low cost scanner (comprised of Microsoft Kinects) was used to obtain point data [23]. The scanner was used in place of a higher resolution commercial alternative because it is part of the intended final system (with a total cost of around $£ 1,500$ ). The errors reported in this study will have to be lowered to acceptable levels using a combination of improvements in software and hardware. Potential hardware improvements come from the calibration method used to obtain extrinsic parameters, and processes which correct distortions in the Kinect's depth information. Future developments in the software algorithm come from increased sophistication. Questions remain regarding how appropriately the Coons patch represents the unseen chest surface beneath the breast. Control over the curvature of this surface would enable it to better match the surrounding chest surface, reducing the error arising from poor point selection. Kovacs et al. [9] refined the breast region based on coincidence between the scanned surface and the Coons patch. Automatic detection of features based on surface curvature or physical palpation would increase reliability of operation and consistency between scans. For example, the IMF is a region of high surface curvature in the majority of cases and an obvious feature to automatically identify.

Following developments in the hardware and software, future studies should assess accuracy and efficacy using human participants. Further advances in software should focus on creating a compiled executable independent of commercial software packages. 


\section{Conclusions}

The presented system represents a method of calculating breast volume using a combination of low-cost scanning hardware (around $£ 1,500$ ) and software. Future developments will work on compiling a software package which is independent of commercial systems.

A systematic over-estimation of breast volume was observed: $130 \mathrm{cc}$ with the $400 \mathrm{~g}$ prosthesis (30.3\%) and $206 \mathrm{cc}(33.3 \%)$ with the $600 \mathrm{~g}$ prosthesis, suggesting positive proportional bias.

Difficulties in assessing absolute breast volume occur from difficulty in identifying an accurate breast boundary, and defining an appropriate chest wall. This error was minimised by placing the breast on a flat surface, with $77 \mathrm{cc}$ over estimation with the $400 \mathrm{~g}$ prostheses (17.9\%) and $62 \mathrm{cc}$ over estimation with the $600 \mathrm{~g}$ prosthesis (10\%).

Reliability of breast measurement varied between operators and was on average, $\pm 59.7 \mathrm{cc}$ for the 400 $\mathrm{g}$ prosthesis (13.9\%) and $\pm 34.7 \mathrm{cc}$ for the $600 \mathrm{~g}$ prosthesis $(5.6 \%)$. This approaches the levels necessary to distinguish between individual implant sizes (around $25-50 \mathrm{cc}$ ). However, the observed proportional error in volume measurement compounds with reliability to make assessments of differential volume (before and after surgery for example) more difficult.

A number of potential improvements in the hardware and software components of this system will make future iterations of this system more accurate, minimising the observed proportional error should be a focus of future work.

\section{References}

1. Hurst CA, Eppley BL, Havlik RJ, Sadove AM (2007) Surgical Cephalometrics: Applications and Developments. Plastic and Reconstructive Surgery 120:92-104.

2. McIntyre GT, Mossey PA (2003) Size and shape measurement in contemporary cephalometrics. The European Journal of Orthodontics 25:231-242.

3. Tepper OM, Unger JG, Small KH, et al. (2010) Mammometrics: the standardization of aesthetic and reconstructive breast surgery. Plastic and reconstructive surgery 125:393-400. doi: 10.1097/PRS.0b013e3181c4966e

4. Liu C, Luan J, Mu L, Ji K (2010) The role of three-dimensional scanning technique in evaluation of breast asymmetry in breast augmentation: a 100-case study. Plastic and Reconstructive Surgery 126:2125-32. doi: 10.1097/PRS.0b013e3181f46ec6

5. Jeevan R, Cromwell D, Browne J, et al. (2010) National Mastectomy and Breast Reconstruction Audit.

6. Oliveira H, Patete P, Baroni G, Cardoso J (2011) Development of a bcct quantitative 3d evaluation system through low-cost solutions. 2nd International Conference on 3D Body Scanning Technologies. pp 25-26

7. Wheat J, Choppin S, Goyal A (2013) Development and Assessment of a Microsoft Kinect based system for imaging the breast in three dimensions. Medical engineering \& physics

8. Catherwood T, McCaughan E, Greer E, et al. (2011) Validation of a passive stereophotogrammetry system for imaging of the breast: a geometric analysis. Medical engineering \& physics 33:900-5. doi: 10.1016/j.medengphy.2011.02.005

9. Kovacs L, Eder M, Hollweck R, et al. (2006) New aspects of breast volume measurement using 3-dimensional surface imaging. Annals of plastic surgery 57:602-10. doi: 10.1097/01.sap.0000235455.21775.6a

10. Kovacs L, Eder M, Hollweck R, et al. (2007) Comparison between breast volume measurement using 3D surface imaging and classical techniques. Breast (Edinburgh, Scotland) 16:137-45. doi: 10.1016/j.breast.2006.08.001

11. Tepper OM, Small K, Rudolph L, et al. (2006) Virtual 3-dimensional modeling as a valuable adjunct to aesthetic and reconstructive breast surgery. American journal of surgery 192:548-51. doi: 10.1016/j.amjsurg.2006.06.026

12. Eder M, Waldenfels $F$ V, Swobodnik A, et al. (2011) Objective breast symmetry evaluation using 3-D surface imaging. Breast (Edinburgh, Scotland) 1-7. doi: 10.1016/j.breast.2011.07.016

13. Losken A, Seify H, Denson DD, et al. (2005) Validating Three-Dimensional Imaging of the Breast. Annals of Plastic Surgery 54:471-476. doi: 10.1097/01.sap.0000155278.87790.a1 
14. Creasman CN, Mordaunt D, Liolios T, et al. (2011) Four-dimensional breast imaging, part I: introduction of a technology-driven, evidence-based approach to breast augmentation planning. Aesthetic surgery journal / the American Society for Aesthetic Plastic surgery 31:914-24. doi: 10.1177/1090820X11423916

15. Henseler H, Khambay BS, Bowman A, et al. (2011) Investigation into accuracy and reproducibility of a 3D breast imaging system using multiple stereo cameras. Journal of plastic, reconstructive \& aesthetic surgery : JPRAS 64:577-82. doi: 10.1016/j.bjps.2010.08.044

16. Oliveira H, Cardoso J (2013) Methods for the Aesthetic Evaluation of Breast Cancer Conservation Treatment: A Technological Review. Current Medical Imaging Reviews 32-46.

17. Coons S. (1967) Surfaces for computer-aided design of space forms.

18. Farin G, Hansford D (1999) Discrete Coons patches. Computer Aided Geometric Design 16:691700. doi: 10.1016/S0167-8396(99)00031-X

19. Arun K, Huang T, Blostein S (1987) Least-squares fitting of two 3-D point sets. IEEE Transactions on pattern Analysis and Machine Intelligence 698-700.

20. Challis J (1995) A procedure for determining rigid body transformation parameters. Journal of Biomechanics 28:

21. Spink D. (2000) NURBS toolbox. http://www.aria.uklinux.net/index.php3.

22. Bulstrode N, Bellamy E, Shrotria S (2001) Breast volume assessment: comparing five different techniques. Breast (Edinburgh, Scotland) 10:117-23. doi: 10.1054/brst.2000.0196

23. Clarkson S, Choppin S, Hart J, et al. (2012) Calculating Body Segment Inertia Parameters from a Single Rapid Scan Using the Microsoft Kinect. 3D Body Scanning. Lugano, Switzerland, pp 153163 